\title{
DESEMPENHO E QUALIDADE DOS OVOS DE POEDEIRAS ALIMENTADAS COM SEMENTE DE URUCUM
}

\author{
Elis Regina de Moraes Garcia ${ }^{1}$ \\ Flavia Kleszcz da Cruz ${ }^{2}$ \\ Rosemary Pereira de Pedro Souza ${ }^{3}$ \\ William Britez Feliciano ${ }^{3}$ \\ Laura Ramos de Ávila ${ }^{3}$ \\ Rosileide Vilalba Rohod ${ }^{4}$
}

GARCIA, E. R. de M.; CRUZ, F. K. da; SOUZA, R. P. de P.; FELICIANO, W. B.; ÁVILA, L. R. de; ROHOD, R. V. Desempenho e qualidade dos ovos de poedeiras alimentadas com semente de urucum. Arq. Ciênc. Vet. Zool. UNIPAR, Umuarama, v.18, n.1, p. 17-20, jan./mar. 2015.

\begin{abstract}
RESUMO: Objetivou-se avaliar o efeito da adição de níveis de semente de urucum moída nas rações sobre o desempenho e a qualidade dos ovos de poedeiras comerciais de ovos vermelhos. Os tratamentos foram constituídos por cinco dietas, sendo quatro experimentais contendo níveis de inclusão de semente de urucum moída $(0,5 ; 1,0 ; 1,5$ e 2,0\%) e um grupo controle. Adotou-se um delineamento inteiramente casualizado, com quatro repetições e oito aves por unidade experimental. As variáveis analisadas foram: consumo de ração, porcentagem de postura, massa de ovos, conversão alimentar ( $\mathrm{kg} / \mathrm{kg}$ e kg/dz), peso médio do ovo, unidade Haugh, índice de gema, cor, gravidade específica, pH de gema e de albúmen, porcentagem e espessura da casca. As variáveis de desempenho e qualidade dos ovos não foram influenciadas $(\mathrm{P}>0,05)$ pelos níveis de semente de urucum estudados, exceto a coloração da gema que apresentou efeito quadrático ( $\left.\mathrm{Y}=5,93+5,86 \mathrm{X}-1,51 \mathrm{X}^{2}\right)$, demonstrando que a coloração da gema crua foi intensificada com a inclusão de 1,94\% de semente de urucum às rações. Conclui-se que adição de semente de urucum moída em níveis de até $2,0 \%$ na ração de poedeiras comerciais de ovos vermelhos não influencia o desempenho e a inclusão de 1,94\% intensifica a coloração da gema.
\end{abstract}

PALAVRAS-CHAVE: Bixa orellana. Consumo de ração. Corantes naturais. Pigmentação. Unidade Haugh.

\section{PERFORMANCE AND QUALITY OF EGGS FROM LAYING HENS FED WITH ANNATTO SEED}

\begin{abstract}
This paper had the aim of evaluating the effect of adding annatto seeds ground in the feeds on the performance and quality of the eggs from red-egg laying hens. The treatments consisted of five diets, four experimental ones containing the inclusion of ground annatto seeds $(0.5,1.0,1.5$ and $2.0 \%)$ and a control group. A completely randomized design was adopted, with four replicates and eight birds per experimental unit. The variables analyzed were feed intake, egg production, egg mass, feed conversion ( $\mathrm{kg} / \mathrm{kg}$ and $\mathrm{kg} / \mathrm{dozen})$, average egg weight, Haugh unit, yolk index, color, specific gravity, $\mathrm{pH}$ yolk and albumen, shell percentage and thickness. The performance variables and egg quality were not affected (P $>0.05)$ by the levels of annatto seed studied, except the color of the yolk, which presented a quadratic effect $\left(Y=5.93+5,86 \mathrm{x}-1,51 \mathrm{x}^{2}\right)$ demonstrating that the staining of the raw egg yolk was enhanced with the addition of $1.94 \%$ annatto seed to the feeds. It can be concluded that the addition of ground annatto seed at levels up to $2.0 \%$ in the feed of red-egg laying hens does not influence the performance, and the inclusion of $1.94 \%$ intensifies the color of the yolk.
\end{abstract}

KEYWORDS: Bixa orellana. Feed intake. Natural dyes. Pigment. Haugh unit.

\section{RENDIMIENTO Y CALIDAD DE LOS HUEVOS DE GALLINAS PONEDORAS ALIMENTADAS CON SEMILLAS DE ACHIOTE}

RESUMEN: Este estudio tuvo como objetivo evaluar el efecto de la adición de niveles de semillas de achiote molidas en piensos, sobre el rendimiento y la calidad de los huevos de gallinas ponedoras de huevos rojos. Los tratamientos se han constituido por cinco dietas, siendo cuatro experimentales conteniendo niveles de inclusión de semillas de achiote molidas $(0,5$, $1,0,1,5$ y $2,0 \%$ ) y un grupo control. Se adoptó un diseño completamente al azar, con cuatro repeticiones y ocho aves por unidad experimental. Las variables analizadas fueron: consumo de alimento, producción de huevos, masa de huevos, conversión alimenticia ( $\mathrm{kg} / \mathrm{kg}$ y kg/dz), peso promedio del huevo, la unidad Haugh, índice de la yema, color, densidad, $\mathrm{pH}$ de yema y de albumen y grosor de la cáscara. Las variables de rendimiento y calidad de los huevos no se vieron afectados $(\mathrm{P}>0,05)$ por los niveles de semillas de achiote estudiados, excepto el color de la yema que mostró un efecto cuadrático $(\mathrm{Y}=5,93+5,86 \mathrm{x}-$ $\left.-1,51 \mathrm{x}^{2}\right)$, lo que demuestra que la tinción de la yema cruda fue intensificada con la adición de 1,94\% de semillas de achiote a los piensos. Se concluye que la adición de semillas de achiote molidas a niveles de hasta 2,0\% en la dieta de las gallinas

DOI: https://doi.org/10.25110/arqvet.v18i1.2015.5364

${ }^{1}$ Professora Adjunta do Curso de Zootecnia e do Programa de Pós-graduação em Zootecnia - Universidade Estadual de Mato Grosso do Sul - Rodovia Aquidauana/UEMS - Km 12 - CEP: 79200-000 - Aquidauana, MS. Brasil. E-mail: ermgarcia@uems.br. Autor para correspondência.

${ }^{2}$ Acadêmica do Programa de Pós-Graduação em Zootecnia (Doutorado) - Universidade Estadual de Maringá, Maringá, PR. E-mail: flaviakleszcz@hotmail. com

${ }^{3}$ Acadêmicos do Curso de Graduação em Zootecnia - Universidade Estadual de Mato Grosso do Sul, Aquidauana, MS. E-mail: rosemaryppsouza@hotmail. com; william_britez@hotmail.com; laura1avila@hotmail.com

${ }^{4}$ Mestre em Zootecnia - Universidade Estadual de Mato Grosso do Sul, Aquidauana, MS. E-mail: rosileid_r@yahoo.com.br 
ponedoras de huevos rojos no influye en el rendimiento y la inclusión de 1,94\% intensifica el color de la yema.

PALABRAS CLAVE: Bixa orellana. Consumo de pienso. Tintes naturales. Pigmentos. Unidad Haugh.

\section{Introdução}

Considerado um alimento completo e de alto valor biológico, o ovo contribui com aproximadamente 20\% das recomendações diárias de proteína (APPLEGATE, 2000), além de ser considerado excelente fonte de colina, nutriente essencial para o bom funcionamento das células (ZEISEL, 2000), gorduras, vitaminas e minerais (PASCOAL et al., 2008).

O atributo cor está correlacionado com a aceitação de um alimento, sendo um componente fundamental de qualidade que, apesar de subjetivo, induz aos apelos de sabor, aroma e textura do alimento. Muitas vezes torna-se necessário adicionar cor aos alimentos para aumentar a atração e estimular o apetite, de forma que se a cor for atraente, apesar da aparência e odor, dificilmente o alimento não será ingerido ou pelo menos provado (SILVA; ALBINO; GODÓI, 2000).

$\mathrm{Na}$ indústria avícola é muito importante o uso de substâncias pigmentantes, já que o consumidor associa a pigmentação da gema do ovo com a quantidade de vitaminas (ARAYA et al., 1977). As xantofilas são um conjunto de carotenoides que fazem parte da constituição do milho e contribuem para a pigmentação, porém, durante a sua estocagem parte dessas xantofilas se perde (HARDER, 2005).

$\mathrm{O}$ alto custo dos pigmentantes sintéticos utilizados, os possíveis efeitos prejudiciais que eles causam à saúde humana e animal, e a necessidade de diversificação agrícola, tem impulsionado as pesquisas na área de nutrição animal a encontrar alternativas para substituir o uso de ingredientes sintéticos por naturais.

Dentre as fontes naturais de pigmentos encontra-se o urucum (Bixa orellana L.), planta nativa, não carcinogênica e atóxica que pode ser encontrada em toda América Central e do Sul, cujo fruto é composto por proteínas, beta-caroteno e outros carotenoides, sendo os mais abundantes a bixina e a norbixina, apresentando grande capacidade pigmentante (BRAZ et al., 2007).

Da produção brasileira $70 \%$ dos grãos produzidos são destinados ao processamento do colorau (corante de uso doméstico), 20\% são utilizados na produção do corante e apenas $10 \%$ é destinada a exportação (BATISTA, 1994). Além disso, devido aos compostos carotenoides presentes em sua composição, o urucum pode apresentar ação antioxidante, protegendo as células de danos oxidativos provocados por radicais livres, podendo assim preservar o alimento, retardando sua deterioração (LOPES et al., 2011).

Diante disso, objetivou-se o avaliar o efeito da adição de níveis de semente de urucum moída nas rações sobre o desempenho e a qualidade dos ovos de poedeiras comerciais de ovos vermelhos.

\section{Material e Métodos}

Foram utilizadas 160 aves da linhagem comercial Dekalb Brown (poedeiras de ovos vermelhos) com 40 semanas de idade, durante o período de 63 dias (três ciclos de 21 dias). As aves foram alojadas duas a duas, em gaiolas de arame galvanizado com quatro divisões de 25 x 40 x $45 \mathrm{~cm}$, em galpão convencional de postura com cobertura de telhas de fibrocimento.

A ração e a água foram fornecidas ad libitum e as aves foram selecionadas por peso (média $\pm 10 \%$ ) e produção de ovos antes do início do experimento, e submetidas a sete dias de adaptação às dietas experimentais. O programa de iluminação adotado foi de 17 horas por dia (iluminação natural + artificial). Durante todo período experimental foram averiguadas as condições térmicas do aviário por meio das temperaturas máxima e mínima e a umidade relativa do ar, correspondendo às médias de $34,9^{\circ} \mathrm{C} \pm 3,1^{\circ} \mathrm{C}, 24,6^{\circ} \mathrm{C} \pm 1,8^{\circ} \mathrm{C}$ e $78,0 \% \pm 11,8 \%$, respectivamente.

Adotou-se um delineamento inteiramente casualizado, com cinco tratamentos, quatro repetições e oito aves por unidade experimental. Os tratamentos foram constituídos por cinco dietas, sendo quatro experimentais contendo níveis de inclusão de semente de urucum moída $(0,5 ; 1,0 ; 1,5$ e $2,0 \%$ ) e um grupo controle.

As aves foram alimentadas diariamente com ração comercial, semente de urucum moída e inerte (amido de milho). A ração comercial utilizada foi elaborada à base de milho, farelo de soja, calcário calcítico e núcleo de forma a atender as exigências nutricionais das aves. Os níveis de semente de urucum adicionados foram calculados com base no peso da ração e ao grupo controle que recebeu somente ração comercial.

Ao $21^{\circ}$ dia de cada ciclo foram avaliadas as características relacionadas ao desempenho zootécnico como consumo de ração (g/ave/dia), porcentagem de postura, massa de ovos (g/ave/dia), conversão alimentar $(\mathrm{kg} / \mathrm{kg}, \mathrm{kg} / \mathrm{dz})$ e peso dos ovos (g).

O consumo de ração foi registrado semanalmente e calculado por meio da diferença entre a quantidade de ração fornecida e a de sobras ao término de cada semana que compôs o ciclo. A produção de ovos foi registrada diariamente com duas coletas diárias. A massa de ovos foi obtida pela multiplicação do peso médio pela percentagem de produção dos ovos.

A conversão alimentar foi calculada por meio do consumo médio de ração $(\mathrm{kg})$ dividido por quilos de ovos (conversão $\mathrm{kg} / \mathrm{kg}$ ) e por dúzia de ovos produzidos (conversão $\mathrm{kg} / \mathrm{dz}$ ).

Nos últimos três dias de cada ciclo, o peso médio e as principais características que expressam a qualidade interna e externa nos ovos foram avaliados. Com base nos dados de peso total e número de ovos da unidade experimental, calculou-se o peso médio dos ovos. A análise de qualidade foi determinada por meio de três ovos íntegros coletados aleatoriamente de cada parcela por dia. Em todos os ovos coletados foi realizada a pesagem unitária por meio de balança semi-analítica $( \pm 0,001 \mathrm{~g})$.

Os ovos inteiros, depois de realizada a pesagem, foram submetidos à análise de gravidade específica. $\mathrm{O}$ valor foi obtido pela imersão dos ovos em baldes plásticos, que continham diferentes concentrações salinas, com densidades que variaram de 1,070 a 1,098 com intervalo de 0,0040. As concentrações das soluções salinas foram ajustadas periodicamente com a utilização de um densímetro de petróleo para 
líquidos. Os ovos foram submersos em baldes da menor até a maior concentração salina, e foram retirados ao flutuarem até a superfície, sendo então anotado o valor da densidade. Posteriormente, realizou-se a quebra dos ovos em superfície plana e lisa de vidro.

Com o auxílio de um paquímetro digital, as medidas de altura do albúmen e da gema foram mensuradas e expressas em mm. Por meio da medida da altura de albúmen (mm) e peso unitário do ovo (g), foram calculados os valores da unidade Haugh determinada pela equação descrita por Silversides e Budgell (2004): $\mathrm{UH}=100 \log \left(\mathrm{H}+7,75-1,7 \mathrm{~W}^{0,37}\right)$, em que, $\mathrm{H}=$ altura do albúmen $(\mathrm{mm})$ e $\mathrm{W}=$ peso do ovo $(\mathrm{g})$. Após, com auxílio de um paquímetro manual $( \pm 0,05 \mathrm{~mm})$, determinou-se o diâmetro da gema, e com base nos valores obtidos, foi mensurado o índice de gema (altura/diâmetro).

Por meio de um leque colorimétrico DSM (Yolk Color Fan $)^{\circledR}$ foi efetuada a análise de coloração da gema crua e com um medidor de $\mathrm{pH}$ de bancada foram obtidos o $\mathrm{pH}$ do albúmen e da gema. Em seguida, as cascas foram lavadas e submetidas à secagem ambiente por 48 horas e pesadas para a determinação da qualidade externa, por meio do peso $(\mathrm{g})$, porcentagem $(\%)$ e espessura da casca $(\mathrm{mm})$.

Os resultados obtidos foram submetidos à análise de variância e os graus de liberdade referentes aos diferentes níveis de urucum $(\mathrm{P}<0,05)$ foram desdobrados em polinômios ortogonais por meio do método dos quadrados mínimos.

\section{Resultados e Discussão}

As variáveis de desempenho não foram influenciadas $(\mathrm{P}>0,05)$ pelos níveis de urucum estudados (Tabela 1$)$. Resultados semelhantes foram encontrados por Braz et al. (2007) e Garcia et al. (2009) que trabalhando com rações à base de sorgo com diferentes níveis de semente residual de urucum $(0,5$ a $2,5 \%)$ e semente de urucum moída $(0,5$ a 2,0\%), respectivamente, na alimentação de poedeiras, não encontraram efeito $(\mathrm{P}>0,05)$ sobre o consumo de ração, massa dos ovos, conversão alimentar e peso dos ovos.

Por outro lado, Silva et al. (2006), ao adicionarem semente residual de urucum $(0,2,4,8$ e $12 \%)$ em dietas para poedeiras com $40 \%$ de sorgo, observaram maior produção de ovos com o aumento da inclusão de urucum. As diferenças de resultados encontrados na literatura e no presente trabalho, provavelmente, estejam relacionadas com os níveis de semente de urucum testados.

Tabela 1: Consumo de ração (CR), porcentagem de postura (PP), massa dos ovos (MO), conversão alimentar (CA) (kg/kg e $\mathrm{kd} / \mathrm{dz}$ ) e peso dos ovos (PO) de poedeiras comerciais de ovos vermelhos alimentadas com níveis de semente de urucum (SU)

\begin{tabular}{ccccccc}
\hline SU $(\%)$ & CR (g/ave/dia) & PP $(\%)$ & MO $(\mathrm{g})$ & CA $(\mathrm{kg} / \mathrm{kg})$ & $\mathrm{CA}(\mathrm{kg} / \mathrm{dz})$ & $\mathrm{PO}(\mathrm{g})$ \\
\hline 0,0 & 104,54 & 87,98 & 52,27 & 2,02 & 1,44 & 59,42 \\
0,5 & 100,50 & 84,11 & 49,88 & 2,04 & 1,45 & 59,23 \\
1,0 & 100,17 & 82,78 & 48,82 & 2,08 & 1,47 & 58,97 \\
1,5 & 101,11 & 88,19 & 53,16 & 1,92 & 1,39 & 60,26 \\
2,0 & 101,55 & 84,44 & 50,91 & 2,01 & 1,45 & 60,25 \\
Média & 101,57 & 85,50 & 51,01 & 2,02 & 1,44 & 59,62 \\
CV (\%) & 2,43 & 4,91 & 4,91 & 5,26 & 5,12 & 2,28 \\
Regressão & $\mathrm{NS}$ & $\mathrm{NS}$ & $\mathrm{NS}$ & $\mathrm{NS}$ & $\mathrm{NS}$ & $\mathrm{NS}$ \\
\hline CV = coeficiente de variação. NS = não significativo. & & &
\end{tabular}

Para a coloração da gema constatou-se efeito quadrático, demonstrando que a cor da gema crua foi intensificada com a inclusão de 1,94\% de semente de urucum às rações das poedeiras (Tabela 2).

A semente de urucum possui substâncias pigmen- tantes (xantofilas e carotenoides) que não são sintetizados pela ave e, quando adicionadas às rações, proporcionam maior intensidade de coloração de gema (ARRAYA et al., 1977), conforme observado no presente estudo.

Tabela 2: Unidade Haugh (UH), índice de gema (IG), coloração de gema (CG), pH do albúmen (pHa) e da gema (pHg), porcentagem de casca (PC), espessura da casca (EC) e gravidade específica (GE) de ovos provenientes de poedeiras comerciais de ovos vermelhos alimentadas com níveis de semente de urucum (SU)

\begin{tabular}{cccccccccc}
\hline SU $(\%)$ & UH & IG $(\mathrm{mm})$ & CG & pHa & pHg & PC & EC (mm) & GE \\
\hline 0,0 & 92,21 & 0,43 & $5,82 \mathrm{e}$ & 7,96 & 6,19 & 9,50 & 0,371 & 1,091 \\
0,5 & 94,76 & 0,43 & $8,73 \mathrm{~d}$ & 7,88 & 6,15 & 9,40 & 0,367 & 1,091 \\
1,0 & 95,39 & 0,43 & $10,20 \mathrm{c}$ & 7,92 & 6,29 & 9,62 & 0,371 & 1,091 \\
1,5 & 92,08 & 0,43 & $11,20 \mathrm{~b}$ & 7,93 & 6,15 & 9,30 & 0,366 & 1,090 \\
2,0 & 95,12 & 0,43 & $11,70 \mathrm{a}$ & 7,90 & 6,18 & 9,33 & 0,365 & 1,090 \\
Média & 93,91 & 0,43 & 9,53 & 7,92 & 6,19 & 9,43 & 0,368 & 1,091 \\
CV (\%) & 3,53 & 2,36 & 1,45 & 1,41 & 1,41 & 2,52 & 2,35 & 0,14 \\
Regressão & $\mathrm{NS}$ & $\mathrm{NS}$ & $\mathrm{Q}$ & $\mathrm{NS}$ & $\mathrm{NS}$ & $\mathrm{NS}$ & $\mathrm{NS}$ & $\mathrm{NS}$ \\
\hline
\end{tabular}

Médias seguidas de letras diferentes na coluna diferem entre si pelo teste Tukey $(\mathrm{P}<0,05) . \mathrm{CV}=$ coeficiente de variação. $\mathrm{Q}=\mathrm{Efeito}$ de regressão quadrático; $\mathrm{NS}=$ não significativo. Coloração da gema: $\mathrm{Y}=5,93+5,86 \mathrm{X}-1,51 \mathrm{X}^{2}, \mathrm{R}^{2}=0,99$ 
Garcia et al. (2009), avaliando os efeitos da inclusão de semente de urucum moída sobre a qualidade dos ovos de poedeiras alimentadas com rações a base de sorgo, observaram que o aumento dos níveis de 0,5 até $2,5 \%$ de semente de urucum moída na dieta melhorou a coloração da gemas dos ovos.

Resultados semelhantes foram encontrados por Braz et al. (2007) que observaram aumento linear da intensidade da coloração da gema ao fornecerem rações para poedeiras baseadas em sorgo e farelo de soja, contendo semente residual de urucum em níveis de até 2,0\%.

Para as demais variáveis relacionadas com a qualidade dos ovos não se observou efeito $(\mathrm{P}>0,05)$ da inclusão de semente de urucum moída às rações, corroborando os relatos de Silva, Albino e Godói (2000), Braz et al. (2007), Carvalho et al. (2009) e Garcia et al (2009), que trabalhando com diferentes produtos e subprodutos do urucum em diferentes níveis de inclusão $(0,2$ até $2,5 \%)$ também não observaram efeito para as variáveis Unidade Haugh, gravidade específica, índice de gema, $\mathrm{pH}$ do albúmen, $\mathrm{pH}$ da gema e porcentagem e espessura de casca.

\section{Conclusão}

Conclui-se que adição de semente de urucum moída em níveis de até 2,0\% na ração de poedeiras comerciais de ovos vermelhos não influencia o desempenho e a inclusão de $1,94 \%$ intensifica a coloração da gema.

\section{Referências}

APPLEGATE, E. Introduction: nutritional and functional roles of eggs in the diet. Journal of the American College of Nutrition, v. 19, p. 495-498, 2000.

ARRAYA, H. H. et al. Composicion y empleo del achiote (B. orellana $L$.) em raciones para gallinas ponedoras, para la pigmentacion de la yema del huevo. Agronomica Costariquense, v. 1, p. 143-150, 1977.

BATISTA, C. L. L. C. Produção e avaliação da estabilidade de corante hidrossolúvel de urucum. Viçosa: UFV, 1994. 71 p.

BRAZ, N. M. et al. Semente residual do urucum na alimentação de poedeiras comerciais: desempenho e características dos ovos. Acta Scientiarum Animal Science, v. 29, p. 129-133, 2007.

CARVALHO, P. R. et al. Supplementation carotenoid compounds derived from seed integral ground annatto (Bixa Orellana L.) in the feed laying hens to produce eggs special. Pakistan Journal of Nutrition, v. 8, p. 1906-1909, 2009.

GARCIA, E. A. et al. Desempenho e qualidade dos ovos de poedeiras comerciais alimentadas com semente de urucum (Bixa Orellana L.) moída na dieta. Veterinária e Zootecnia, v. 16, p. 689-697, 2009.

HARDER, M. N. C. Efeito do urucum (Bixa orellana) na alteração de características de ovos de galinhas poedeiras. 2005. 74 f. Dissertação (Mestrado) - Escola Superior de Agricultura Luiz de Queiroz, Piracicaba, 2005.

LOPES, I. R. V. et al. Desempenho e qualidade dos ovos de poedeiras comerciais alimentadas com rações contendo farelo de coco tratado ou não com antioxidante. Revista Brasileira de Zootecnia, v. 40, p. 2431-2438, 2011.

PASCOAL, L. A. F. et al. Qualidade de ovos comercializados em diferentes estabelecimentos na cidade de Imperatriz-MA. Revista Brasileira de Saúde e Produção Animal, v. 9, p. 150- 157, 2008.

SILVA, J. H. V.; ALBINO, L. F. T.; GODÓI, M. J. S. Efeito do extrato de urucum na pigmentação da gema de ovos. Revista Brasileira de Zootecnia, v. 29, p. 1435-1439, 2000 .

SILVA, J. H. V. et al. Resíduo da semente de urucum (Bixa orellana L.) como corante da gema, pele, bico e ovário de poedeiras avaliado por dois métodos analíticos. Ciência e Agrotecnologia, v. 30, p. 988-994, 2006.

SILVERSIDES, F. G.; BUDGELL, K. The relationships among measures of egg albumen height, $\mathrm{pH}$, and whipping volume. Poultry Science, v. 83, p. 1619-1623, 2004.

ZEISEL, S. H. Choline: needed for normal development of memory. Journal of the American College of Nutrition, v. 19 , p. 528-531, 2000. 